\title{
Local Structural Analysis of Graphitic Carbon Nitrides
}

\author{
Diane M. Haiber ${ }^{1}$, Michael M.J. Treacy ${ }^{2}$ and Peter A. Crozier ${ }^{1}$ \\ 1. School for Engineering of Matter, Transport \& Energy, Arizona State University, Tempe, Arizona \\ 85287-6106, USA \\ 2. Department of Physics, Arizona State University, Tempe, Arizona 85287-6106, USA
}

Graphitic carbon nitrides are layered, polymeric compounds that are widely studied as a semiconducting support for visible-light driven photocatalytic water splitting [1]. However, structural disorder in these materials is poorly understood due to the difficulty of performing high spatial resolution characterization through transmission electron microscopy (TEM) on these beam-sensitive materials. Fully condensed g$\mathrm{C}_{3} \mathrm{~N}_{4}$ contains hexagonally periodic heptazine $\left(\mathrm{C}_{6} \mathrm{~N}_{7}\right)$ or triazine $\left(\mathrm{C}_{3} \mathrm{~N}_{3}\right)$ motifs, bridged by 3 -fold coordinated $\mathrm{N}$-atoms, creating a graphite-like structure with regularly-spaced voids. Synthesis involves calcination of N-rich precursors to yield yellow powders with varying optical absorption. In this way, g$\mathrm{CN}_{\mathrm{x}} \mathrm{H}_{\mathrm{y}}$ compounds are formed with a sub-stoichiometric $\mathrm{C} / \mathrm{N}$ ratio at the expense of residual hydrogen preventing complete structural condensation. In 2007, nuclear magnetic resonance and electron diffraction were combined to solve the average in-plane structure of a $\mathrm{g}-\mathrm{CN}_{\mathrm{x}} \mathrm{H}_{\mathrm{y}}$ compound that consisted of amine $\left(\mathrm{N}-\mathrm{H}_{\mathrm{x}}\right)$ bridged heptazine molecules arranged in zig-zag chains [2]. Based on $\mathrm{x}$-ray and neutron diffraction, the bulk stacking order was determined giving a 3D structural description [3]. Low-dose TEM may be applied to directly image variations of the in-plane structure and obtain a more precise description of structural disorder.

Using an aberration-corrected TEM operating at 300-kV under low dose rate conditions (e.g. $\sim 20 \mathrm{e}^{-}$ $/ \AA^{2} / \mathrm{s}$ ) coupled with a direct electron detector, we have acquired images from three $\mathrm{g}-\mathrm{CN}_{\mathrm{x}} \mathrm{H}_{\mathrm{y}}$ specimens with different degrees of presumed structural disorder. Figure 1(a)-(c) compares TEM images of g$\mathrm{CN}: 1, \mathrm{~g}-\mathrm{CN}: 2$, and $\mathrm{g}-\mathrm{CN}: 3$, respectively, where the suffix refers to the assumed degree of structural disorder ( $\mathrm{g}-\mathrm{CN}$ :3 being the most disordered). Image Fourier transforms (FT's) taken over a large fieldof-view of each compound oriented with the basal planes approximately perpendicular to the beam are displayed in Figure 1(d)-(f). While the image FT for g-CN:1 displays a pseudo-hexagonal symmetry, both $\mathrm{g}-\mathrm{CN}: 2$ and $\mathrm{g}-\mathrm{CN}: 3$ display continuous rings of different diffuseness. Figure 1(g) compares the azimuthally-averaged FT's confirming that each material possesses the same d-spacings but with different degree of broadening suggesting increasing structural disorder.

So far, several techniques are being considered to elucidate in-plane disorder based on TEM imaging. Scanned FT's are being used to generate mean and variance information, similar to fluctuation electron microscopy approaches used for quantification of structural disorder [4]. Figure 2(a) compares the mean and normalized variance generated from scanned FT's (window size $\left.=(6.5 \mathrm{~nm})^{2}\right)$ from each material. The increase in variance observed for g-CN:1 may indicate increasing in-plane order. On the other hand, scanned autocorrelations are employed to produce averaged pseudo radial distribution functions (RDF's) as shown in Figure 2(b). Peaks in the RDF correspond to real-space distances, some of which are annotated in the inset of Figure 2(b). Ideally, structural regularity in the heptazine chain separation distance may be assessed using scanned RDF's and related to disorder.

References:

[1] X. Wang et al. Nat. Mater. 2009, 9, 76-80. 
[2] B.V. Lotsch et al. Chem. Eur. J. 2007, 13, 4969-80.

[3] F. Fina et al. Chem. Mater. 2015, 27, 2612-18.

[4] M.M.J. Treacy et al. Rep. Prog. Phys. 2005, 68, 2899-944.

[5] Support from the U.S. DOE (DE-SC0004954, use of AC-TEM within the LeRoy Eyring Center for Solid State Science at ASU, and use of the K2-IS detector courtesy of Gatan are gratefully acknowledged.
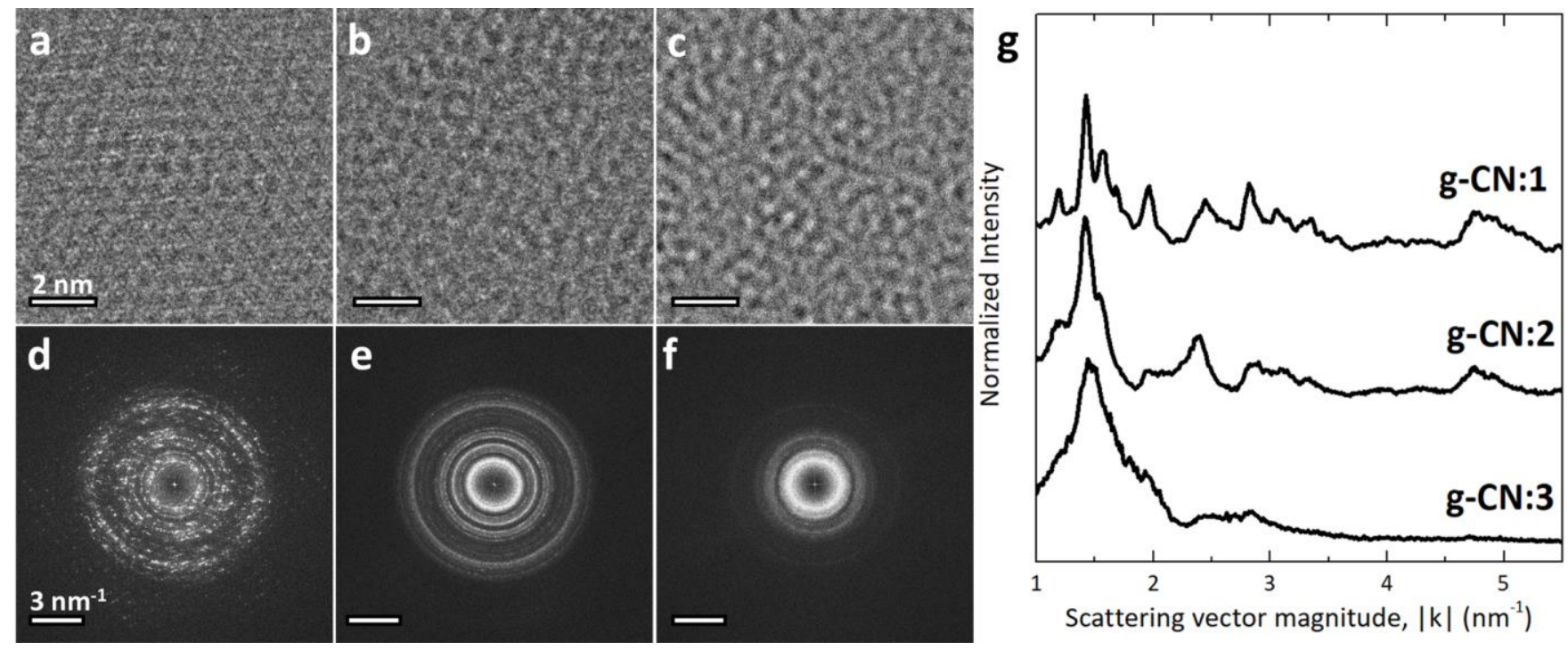

Figure 1. Low dose rate TEM images of (a) g-CN:1, (b) g-CN:2, and (c) g-CN:3 taken from large fieldof-view images predominately oriented perpendicular to the graphitic planes. Corresponding image Fourier transforms (FT's) for (d) g-CN:1, (e) g-CN:2, and (f) g-CN:3. (g) Comparison of azimuthallyaveraged FT's of each sample.
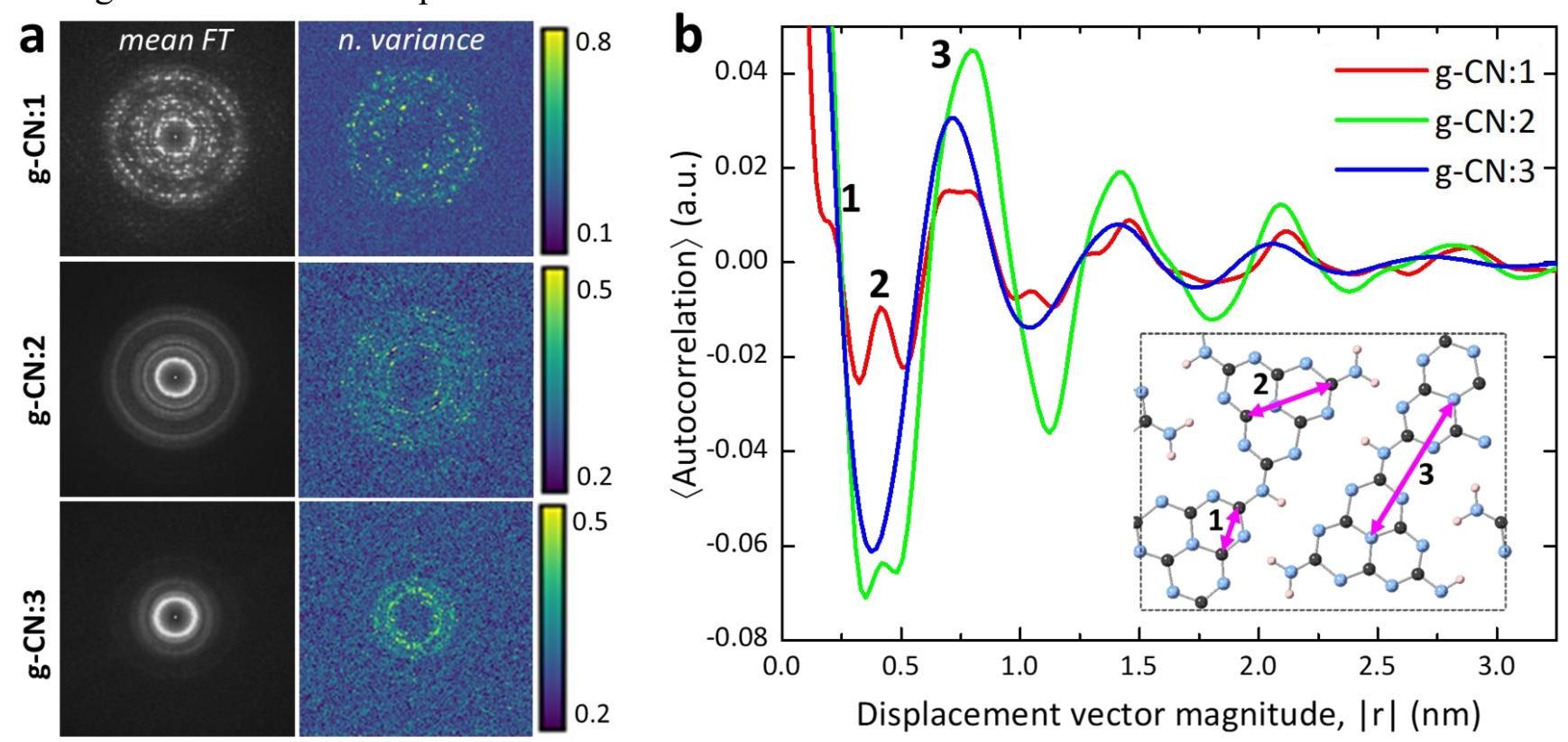

Figure 2. (a) Mean and normalized variance of scanned FT's, taken over regions of $(6.5 \mathrm{~nm})^{2}$ for each g-CN material. (b) Comparison of azimuthally-averaged mean autocorrelation for each g-CN, generated from sets of scanned autocorrelations, also taken with a window size of $(6.5 \mathrm{~nm})^{2}$. The numbered peaks in (b) correspond to different real-space distances, which are depicted in the structure model (inset). 